\title{
Association of High Serum Chemerin with Bone Mineral Density Loss and Osteoporotic Fracture in Elderly Chinese Women
}

\author{
Xi-Yuan Jiang ${ }^{1,2}$, Qing Wang', Ying Zhang ${ }^{3}$, Yong Chen', Long-Fei $\mathrm{Wu}^{4}$ \\ 'Center of Osteoporosis, Kunshan Hospital of Traditional Chinese Medicine, Kunshan, Jiangsu, 215300, People's Republic of China; ${ }^{2}$ School of \\ Medicine, Southeast University, Nanjing, Jiangsu, 210009, People's Republic of China; ${ }^{3}$ Department of Clinical Laboratory, Kunshan Hospital of \\ Traditional Chinese Medicine, Kunshan, Jiangsu, 215300, People's Republic of China; ${ }^{4}$ School of Public Health, Medical College of Soochow University, \\ Suzhou, Jiangsu, 215I23, People's Republic of China
}

Correspondence: Xi-Yuan Jiang, Center of Osteoporosis, Kunshan Hospital of Traditional Chinese Medicine, Kunshan, Jiangsu, 215300, People's Republic of China, Tel/Fax +86-5I2-57928I40, Email 397365264@qq.com

Background: Chemerin has been suggested to be a risk factor for osteoporosis; however, its relationship with osteoporotic fracture is poorly understood. Herein, we intend to explore the association between serum chemerin and osteoporotic fracture.

Methods: A total of 111 elderly women patients diagnosed with osteoporotic fracture were selected as the observation group, and 40 healthy subjects were enrolled as controls. Dual-energy X-ray absorptiometry, enzyme-linked immunosorbent assay, electrochemiluminescence immunoassay, and biochemical analysis were separately performed to determine body bone mineral density (BMD), chemerin levels, bone turnover markers, and other parameters. Pearson's correlation analysis was conducted to examine a relationship between chemerin and laboratory parameters. Moreover, the levels of chemokine-like receptor $1(C M K L R)$, C-C motif chemokine receptor-like 2 (CCRL2), collagen type I alpha (COLA1), and runt-related transcription factor-2 (RUNX2) were confirmed by quantitative polymerase chain reaction, and the effect of chemerin on osteogenic differentiation of hFOB1.19 cells was indicated by tartrate-resistant acid phosphatase and alkaline phosphatase double staining.

Results: A higher level of chemerin was generally detected in patients with osteoporotic fracture compared with those without $(P<0.05)$. Compared with controls, lower BMD levels and higher $\beta$-CTx and P1NP levels were detected in patients with osteoporotic fracture (all $P<0.05)$. Interestingly, chemerin level was negatively correlated to BMD, but positively related to P1NP and $\beta$-CTx. Risk of osteoporotic fracture was 2.75-fold higher in subjects with each standard deviation increment of chemerin. Compared with controls, there were no significant differences in CMKLR1 and CCRL2 mRNA after incubation with osteogenic differentiation medium (all $P>0.05$ ), whereas there was a remarkable decrease of COLA1 and $R U N X 2$ after incubation with chemerin for nine days (all $P<0.05$ ). Furthermore, prolonged incubation with chemerin enhanced osteoclast differentiation and maturation, consequently contributing to an increased risk of fracture. Conclusion: Chemerin is a strong and independent risk factor for osteoporosis-related fracture among elderly Chinese women.

Keywords: chemerin, osteoporotic fracture, risk factor, elderly women

\section{Introduction}

Osteoporosis is a disease adversely affecting the bone health and consequently leading to an increased risk of bone fractures, ${ }^{1}$ in which hip fracture is recognized as the most serious consequence of osteoporosis and osteoporotic vertebral compression fracture is the most common type of osteoporotic fracture worldwide. ${ }^{2}$ With the aging global population accelerating, the incidence of osteoporotic fractures markedly increased in the elderly, with a high resultant morbidity and mortality in the elderly population. Nowadays, it is becoming a serious public health concern worldwide, ${ }^{3}$ particularly in Asia. ${ }^{4}$ Osteoporosis is characterized by decreased bone strength and low bone mass, therefore its risk prediction is largely based on the baseline of bone mineral density (BMD). ${ }^{5}$

Although regional and social differences could be observed in the frequency of osteoporotic fracture and BMD values, ${ }^{6}$ there was a relationship between BMD value and osteoporotic fracture. ${ }^{7,8}$ Compared with BMD alone, ${ }^{9}$ its combination with 
other powerful risk factors could improve the identification accuracy for individuals at high fracture risk. ${ }^{10-12}$ It is therefore necessary to explore additional biomarkers that can improve fracture risk prediction independent of, or combined with, BMD.

Adipocytes and osteoblasts secrete various adipose tissue-derived adipokines, which regulate bone formation and resorption. ${ }^{13,14}$ As a recently discovered adipocyte, chemerin had a cross-sectional association with bone health, ${ }^{15,16}$ and it was also a protein ligand for the $\mathrm{G}$ protein-coupled receptor chemokine-like receptor 1 (CMKLR1) that modulated adipogenesis via binding to $\mathrm{C}-\mathrm{C}$ motif chemokine receptor like 2 (CCRL2) and G-protein-coupled receptor $1,{ }^{17}$ but the pharmacology and signaling properties of these chemerin receptors have been much less characterized. ${ }^{18}$ Using rat model, inhibiting chemerin activity increased osteoblast marker gene expression and mineralization in the bone marrow stromal cells, ${ }^{19}$ and the chemerin was also involved in bone resorption via regulating hematopoietic stem cell osteoclastogenesis. ${ }^{20}$ Moreover, there were associations between circulating chemerin levels and BMD or osteoporosis in humans. ${ }^{16,19,21-23} \mathrm{Up}$ to now, the effect of chemerin on bone metabolism has been verified both in animal models and human population, however, the possibility of chemerin as a useful biomarker for detecting the osteoporotic fracture is still unclear. Consequently, the present study intends to clarify the potential role of serum chemerin in forecasting the occurrence of osteoporotic fracture, and further attempts to elucidate the possible biological mechanism underlying the effect of chemerin on bone metabolism.

\section{Materials and Methods}

\section{Study Subjects}

All the participants aged more than 60 years who were admitted to Center of Osteoporosis at Kunshan Hospital of Traditional Chinese Medicine between January 2019 and December 2020. Patients recently diagnosed with osteoporotic fracture were eligible, and those involved in the final study following the inclusion and exclusion criteria. The process flowchart for analyses was presented in Figure 1. Simultaneously, 40 age- and BMI-matched healthy subjects were recruited as controls. Demographic information such as, age, disease history, and medication use history, was obtained via a detailed questionnaire and physical examination. Samples were obtained from the patients within 1 5 days after fracture and from healthy control subjects at the same time, and all protocols were approved by the Ethics Committee of Kunshan Hospital of Traditional Chinese Medicine (ks1736). Prior to the study, written informed consent was obtained from all individual participants in accordance with the Declaration of Helsinki.

\section{BMD and Laboratory Measurements}

For each individual, BMD $\left(\mathrm{g} / \mathrm{cm}^{2}\right)$ was conducted at the lumbar spine (L1-L4) and femoral neck in the anterior-posterior projection by dual-energy X-ray absorptiometry (DXA; Lunar iDXA, GE Healthcare), during which a well-trained and competent laboratory technologist ensured the accurate and reliable results of all DXA scans. Meanwhile, quality assurance scans were performed daily by scanning an aluminum spine phantom according to the manufacturer's instructions.

To minimize intra-day variations in bone markers, blood samples were drawn from median cubital vein in the morning, allowed to stand for $10 \mathrm{~min}$, and subsequently centrifuged at $3000 \mathrm{rpm}$ for $15 \mathrm{~min}$ to separate serum. Then, serum levels of chemerin were determined using a commercially available enzyme linked immunosorbent assay (ELISA) kit according to manufacturer's instruction (Uscn Life Science Inc., Wuhan, China). Bone resorption ( $\beta$-cross-linked C-terminal cross-linked telopeptide of type I collagen, $\beta$-CTx), bone formation (procollagen type 1 amino-terminal propeptide, P1NP), and 25hydroxyvitamin $\mathrm{D}(25(\mathrm{OH}) \mathrm{D})$ were measured by electrochemiluminescence immunoassay on a cobas 8000 e602 apparatus (Roche Diagnostics) using Roche Elecsys reagent kit. In addition, concentrations of other laboratory parameters including calcium and phosphorus were measured on an automatic biochemical analyzer (cobas E701, Roche Diagnostics).

\section{Cell Culture}

Human fetal osteoblastic cell line (hFOB1.19) obtained from American Type Culture Collection (ATCC, USA) was routinely cultured in the complete medium consisting of 1:1 (w/w) Dulbecco's Modified Eagle's Medium and Ham's F12 Medium (DMEM/F12, Life Technologies), which were supplemented with 10\% fetal bovine serum (Invitrogen) and 


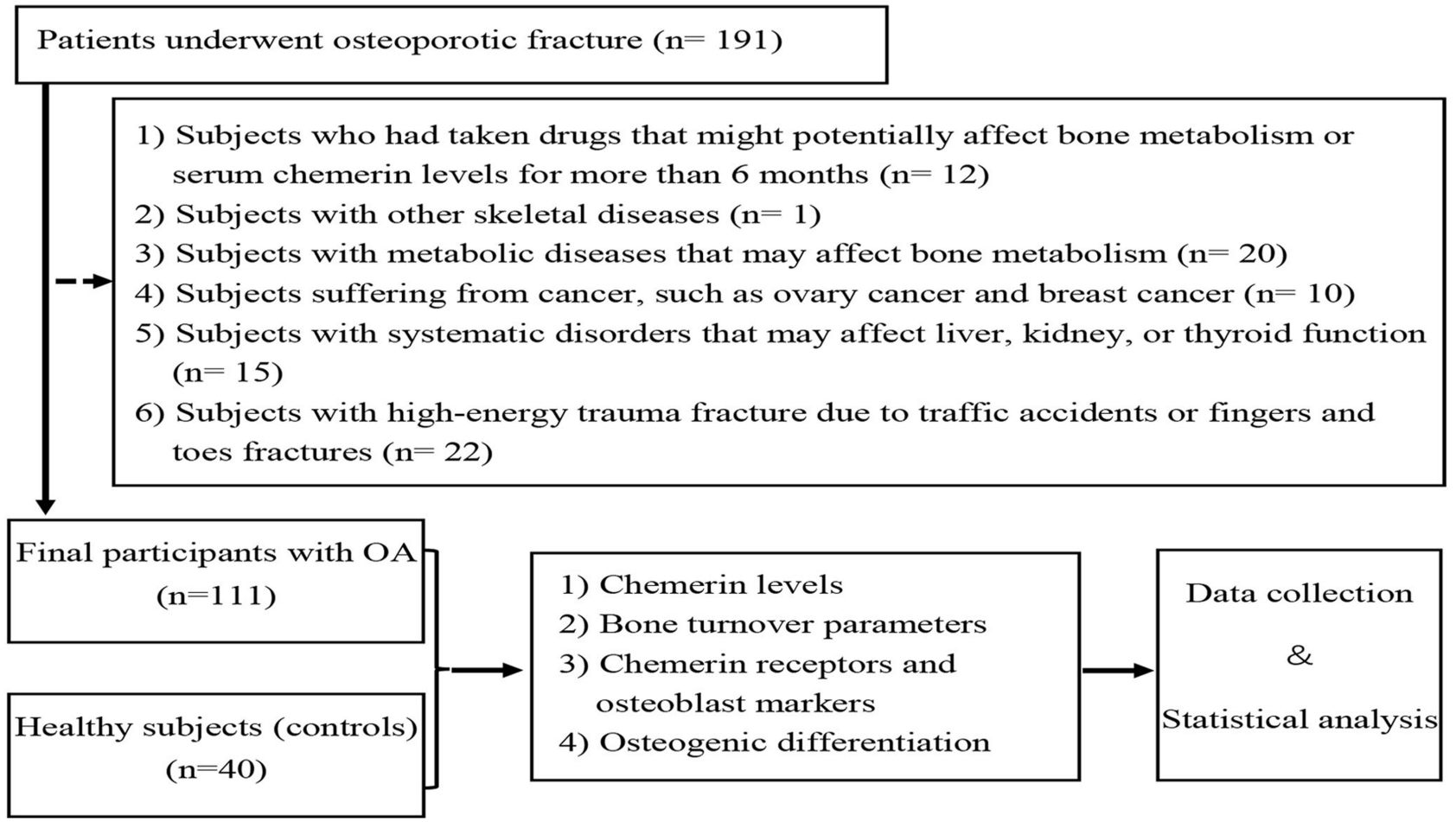

Figure I The process flowchart for analyses of patients with osteoporotic fracture.

$0.3 \mathrm{mg} / \mathrm{mL}$ neomycin (G418, Sigma, St Louis, MO). The cells were placed in an incubator at $34^{\circ} \mathrm{C}$ under an atmosphere of $5 \% \mathrm{CO}_{2}$ until they were fully confluent.

\section{Osteogenic Differentiation}

For in vitro osteogenic differentiation, hFOB1.19 cells were maintained at $34^{\circ} \mathrm{C}$ in the complete medium supplemented with $10 \mathrm{mM}$ glycerol phosphate, $0.25 \mathrm{mM}$ ascorbic acid, and $0.1 \mu \mathrm{M}$ dexamethasone (Sigma-Aldrich).

\section{Quantitative Real-time PCR (qRT-PCR) Analysis}

Total RNA was extracted with TRIzol (Invitrogen, Life Technologies), and then $1 \mu \mathrm{g}$ RNA was used for cDNA synthesis using HiScript SuperMix cDNA Synthesis Kit (Vazyme Biotech, Nanjing, China). Quantitative PCR analysis was performed in duplicates to analyze the levels of chemerin receptors (CMKLR1 and CCRL2) or osteoblast markers (COLA1 and RUNX2) on the automated thermocycler (ABI 9600, Applied Biosystems, Foster City, CA, USA) using Universal SYBR qPCR Master Mix (Vazyme Biotech). Specific mRNA levels of hFOB1.19 cells were normalized to $G A P D H$, and the relative quantification was calculated using the $\triangle \triangle \mathrm{Ct}$ method as specified by the manufacturer.

\section{Alkaline Phosphatase (ALP) Staining Assay}

Human fetal osteoblastic (hFOB1.19) cells were cultured in the osteogenic inductive medium supplemented with human recombinant chemerin (Ca. No. 2324-cm-025, R\&D Systems). After incubation for different times, the cells rinsed twice with phosphate buffered saline were fixed with fixation buffer (Cat. No. 554655). Thereafter, the slides were washed with distilled water and then stained to observe the reddish-purple for active region of acid phosphatase and bluish brown for ALP using a tartrate-resistant acid phosphatase (TRACP) and ALP double-stain kit (Cat. No.64-19-7; Takara Bio. Inc., Shiga, Japan).

\section{Statistical Analysis}

All data were analyzed by the SPSS statistic software 20.0 (IBM Corporation, Armonk, NY, USA) and presented as mean values and standard deviations. The independent $t$-test was performed for comparison of continuous variables between 
two groups, and an analysis of covariance was performed to compare the difference between groups. Pearson's correlation analysis was carried out to evaluate the associations of serum chemerin with other variables. Multiple logistic regression analyses were conducted to generate odds ratios and $95 \%$ confidence interval (CI). $P$-values less than 0.05 were defined as statistically significant.

\section{Results}

\section{Baseline Characteristics of Individuals}

Following the application of inclusion and exclusion criteria, 111 elderly women patients recently diagnosed with osteoporotic fracture were included in the final analyses, among which there were 12 cases of patellofemoral joint fracture and 99 cases of osteoporotic vertebral compression fractures, and there was a significant difference in the incidence of fractures between the two groups $(P=0.001)$. Compared with healthy subjects, the levels of BMD at lumbar spine and femoral neck were lower, whereas those of $\beta$-CTx and P1NP were higher in patients with osteoporotic fracture (all $P<0.05$ ). In addition, there were no statistical differences in other measured laboratory parameters and demographic parameters between the osteoporotic fracture group and the healthy control group (Table 1).

\section{Association of Serum Chemerin Level with BMD and Biochemical Parameters}

As shown in Table 2, the serum chemerin level was inversely correlated to BMD both at lumbar spine and femoral neck, whereas it was positively related to P1NP and $\beta$-CTx (all $P<0.05$ ). In addition, there were no remarkable associations with other parameters (all $P>0.05$ ).

Table I Demographic and Laboratory Characteristics of Participants

\begin{tabular}{|c|c|c|c|c|}
\hline Variables & $\begin{array}{l}\text { Healthy Control Group } \\
\qquad(n=40)\end{array}$ & $\begin{array}{l}\text { Osteoporotic Fracture Group } \\
\qquad(\mathrm{n}=\mid I I)\end{array}$ & $\boldsymbol{t}$ & $P$-value \\
\hline Age (years) & $69.1 \pm 2.4$ & $70.2 \pm 5.7$ & -1.675 & 0.096 \\
\hline Height (cm) & $167.0 \pm 7.4$ & $165.0 \pm 6.1$ & 0.987 & 0.327 \\
\hline Weight (kg) & $57.8 \pm 5.1$ & $55.2 \pm 6.9$ & 1.932 & 0.057 \\
\hline BMI $\left(\mathrm{kg} / \mathrm{m}^{2}\right)$ & $23.63 \pm 2.41$ & $22.77 \pm 2.75$ & 1.731 & 0.085 \\
\hline BMD (lumbar spine) $\left(\mathrm{g} / \mathrm{cm}^{2}\right)$ & $1.096 \pm 0.132$ & $0.852 \pm 0.165$ & 8.447 & 0.000 \\
\hline BMD (femoral neck) $\left(\mathrm{g} / \mathrm{cm}^{2}\right)$ & $0.894 \pm 0.093$ & $0.746 \pm 0.088 *$ & 8.924 & 0.000 \\
\hline PINP (ng/mL) & $56.34 \pm 11.23$ & $71.94 \pm 19.26 *$ & -6.124 & 0.000 \\
\hline$\beta-C T \times(n g / m L)$ & $0.606 \pm 0.129$ & $0.712 \pm 0.249 *$ & -3.381 & 0.001 \\
\hline Calcium (mM) & $2.248 \pm 0.100$ & $2.211 \pm 0.112$ & 0.184 & 0.068 \\
\hline Phosphorus (mM) & $1.175 \pm 0.135$ & $1.152 \pm 0.203$ & 0.799 & 0.426 \\
\hline 25(OH)D (ng/mL) & $15.65 \pm 3.73$ & $15.14 \pm 5.34$ & 0.552 & 0.582 \\
\hline Glucose (mM) & $5.84 \pm 0.50$ & $6.16 \pm 0.60$ & -1.593 & 0.115 \\
\hline Total cholesterol (mM) & $4.02 \pm 0.49$ & $3.96 \pm 0.57$ & 0.504 & 0.615 \\
\hline Triglyceride (mM) & $1.58 \pm 0.47$ & $1.47 \pm 0.58$ & 0.950 & 0.345 \\
\hline
\end{tabular}

Notes: All data were presented as mean \pm standard deviation. $* P<0.01$, compared with healthy controls.

Abbreviations: BMI, body mass index; BMD, bone mineral density; $25(\mathrm{OH}) \mathrm{D}$, 25 -hydroxyvitamin $\mathrm{D}$; PINP, procollagen type I amino-terminal propeptide; $\beta$-CTx, $\beta$ - crosslinked C-terminal telopeptide of type I collagen. 
Table 2 Bivariate Correlation Analyses Between Chemerin Level and Laboratory Parameters

\begin{tabular}{|l|c|c|}
\hline Parameter & $\mathbf{r}$ & P-value \\
\hline Age & 0.147 & 0.072 \\
\hline BMI & -0.051 & 0.538 \\
\hline BMD (lumbar spine) & -0.460 & 0.001 \\
\hline BMD (femoral neck) & -0.403 & 0.001 \\
\hline Calcium & -0.151 & 0.159 \\
\hline Phosphorus & 0.023 & 0.780 \\
\hline $25(O H) D$ & -0.022 & 0.785 \\
\hline PINP & 0.191 & 0.019 \\
\hline$\beta$-CTx & 0.236 & 0.003 \\
\hline Blood glucose & 0.135 & 0.392 \\
\hline Total cholesterol & -0.07 & 0.658 \\
\hline Triglyceride & -0.25 & 0.074 \\
\hline
\end{tabular}

Abbreviations: BMI, body mass index; BMD, bone mineral density; PINP, procollagen type I amino-terminal propeptide; $\beta$-CTx, $\beta$-isomerization of C-terminal telopeptide of type I collagen.

\section{Association of Chemerin Level with the Risk for Osteoporotic Fracture}

As shown in Figure 2, serum chemerin level was significantly higher in patients with osteoporotic fracture compared with healthy controls $(P=0.001)$. To control the confounding effects, we adopted the analysis of covariance and further found that there was a significant difference in the chemerin level of patients between osteoporotic fracture group and healthy control group, even after statistically adjusting for age and BMI $(P=0.038)$. Therefore, these results raised a possibility that serum chemerin could be a more useful biomarker for prediction of osteoporotic fracture. Subsequently, we performed logistic regression analyses and the results revealed that each standard deviation increment of chemerin level was significantly associated with 2.75 -fold higher odds ratios for the patients with osteoporotic fracture after statistically adjusting BMI and age (95\%CI, 1.11 6.85, $P<0.05)$.

\section{Effects of Chemerin on the Human Osteoblastic Cell Differentiation}

To study the potential mechanism of the relationship between serum chemerin levels and osteoporotic fracture, we firstly determined the chemerin transcription level by qRT-PCR in the hFOB1.19 cells cultured with osteogenic medium. Compared with undifferentiated cells, the transcription level of chemerin decreased significantly in a time-dependent manner (Figure 3A). Conversely, when compared with control group, there were no significant differences in the transcription levels of chemerin receptor CMKLR1 and CCRL2 in hFOB1.19 cells after incubation with osteogenic differentiation medium (Figure 3B).

To further explore the effects of chemerin on osteogenic differentiation, the hFOB1.19 cells were incubated with recombinant human chemerin, and then relative mRNA levels of osteoblast marker genes were examined by qRT-PCR. As shown in Figure 4, transcription levels of osteoblast marker genes, COLA1 and RUNX2, increased significantly in the hFOB1.19 cells incubated with osteogenic differentiation medium compared with normal medium. Of note, compared with the control group, a significant decrease of these osteoblast marker genes was detected in the hFOB1.19 cells after incubation with chemerin for nine days.

Lastly, we examined the effect of chemerin on osteogenic differentiation indicated by TRACP\&ALP double staining. Consistently, compared with control group, reprehensive images of hFOB1.19 cells with bluish brown were observed 


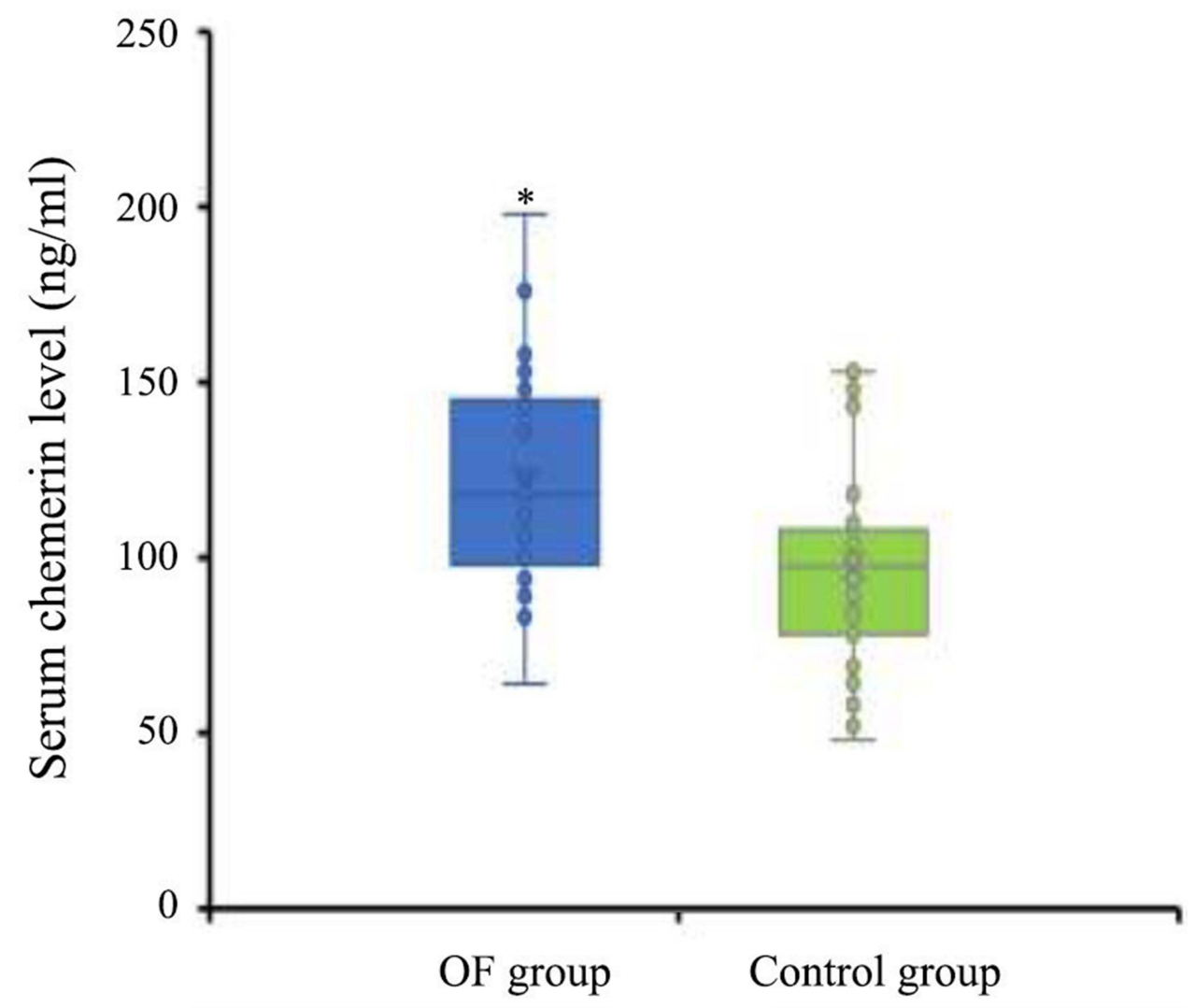

Figure 2 Serum chemerin levels in the patients with osteoporotic fracture and healthy controls determined by ELISA Notes: Data were presented as mean \pm standard deviation. $* P<0.001$, compared with control group.

Abbreviations: OF, osteoporotic fracture; ELISA, enzyme linked immunosorbent assay.

under optical microscope (Figure 5A-D) and a remarkably increased gray value of hFOB1.19 cells was detected in a time-dependent manner (Figure 5E), but no obvious wine red for active region of acid phosphatase was observed after incubation with chemerin for nine days (Figure 5A-D). During osteogenic differentiation, a number of TRAP-positive cells (wine red) with large volume and multiple nuclei were observed in hFOB1.19 cells after incubation with chemerin for 14 days, whereas the bluish brown value of hFOB1.19 cells was lower compared with nine days, suggesting that long incubation with chemerin enhances osteoclast differentiation and maturation.

\section{Discussion}

This is a clinical trial, which explored the association between serum chemerin and osteoporotic fracture. To the best of our knowledge, this is the first study to report a relationship of circulating chemerin with BMD loss and osteoporotic fracture, suggesting that serum chemerin might be a potential biomarker of fracture in the elderly women. In the current study, there were 111 elderly women patients recently diagnosed with osteoporotic fracture in the final study, with 12 cases of patellofemoral joint fracture and 99 cases of osteoporotic vertebral compression fracture. To maintain healthy bone tissue homeostasis, the human body needs enough calcium, phosphorus and calcium-phosphorus metabolismrelated hormones. As reported in a previous study, fracture risk might be increased in individuals with extremely low calcium intake, however a meta-analysis of observational studies relating calcium intake to fracture risk failed to show any association between calcium and hip fracture. ${ }^{24}$ The active hormonal form of vitamin D was a principal regulator of calcium and phosphorus homeostasis through actions on intestine, ${ }^{25}$ and resorption of calcium from bones, consequently determining the amount of renal excretion of these substances. Compared with healthy controls, the serum calcium and $25(\mathrm{OH}) \mathrm{D}$ levels in the current study were slightly lower, whereas the phosphorus levels were mildly higher in patients with osteoporotic fracture, but the difference did not reach statistical significance. In addition, vitamin D insufficiency 

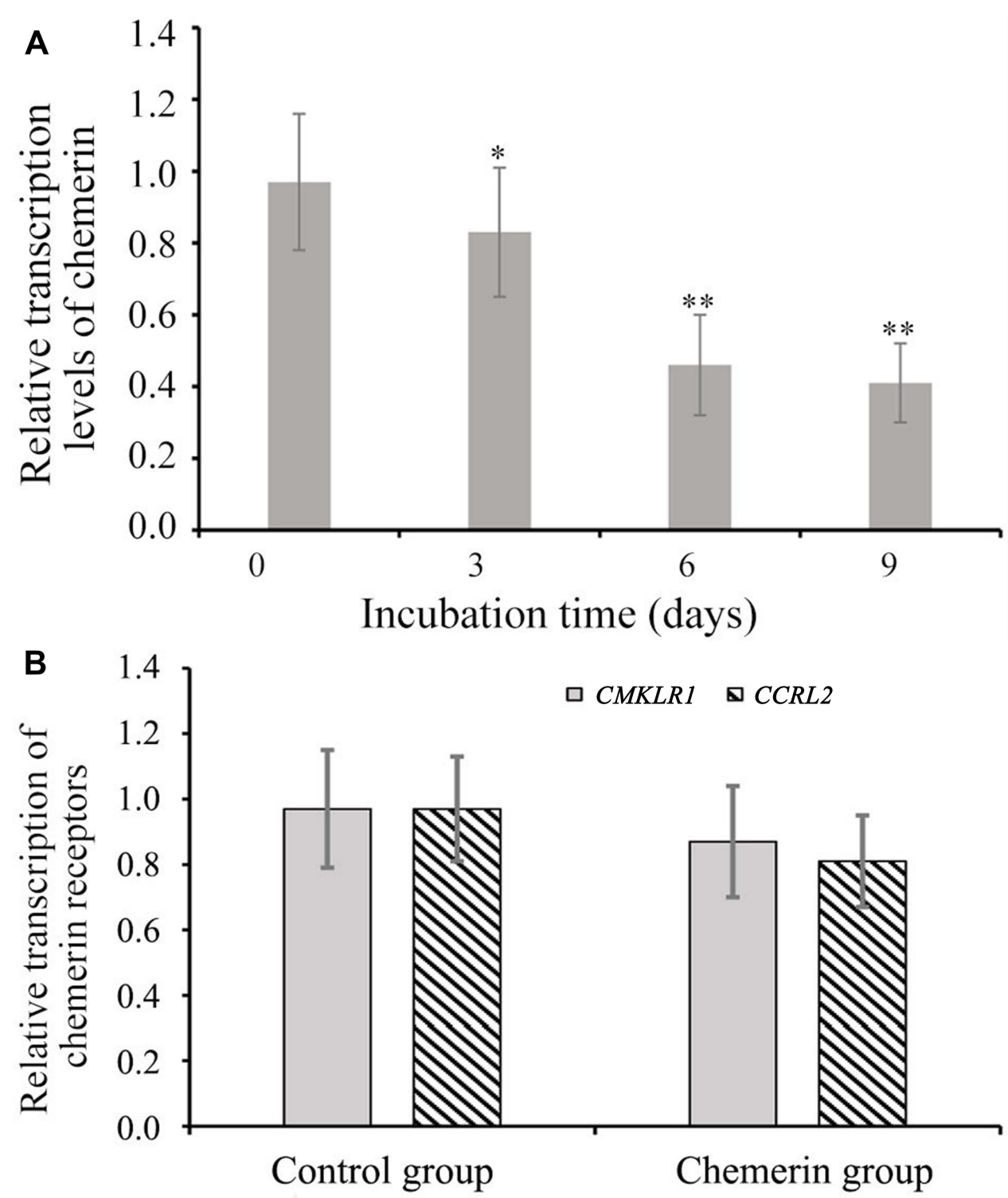

Figure 3 Relative transcription levels of chemerin (A) and its receptor CMKLRI and CCRL2 mRNA (B) in hFOBI.I9 cells analyzed by qRT-PCR.

Notes: (A) hFOBI.19 cells were cultured in the osteogenic differentiation medium for 3,6 , and 9 days and then the transcription level of chemerin was examined by qRTPCR. (B) the transcription levels of chemerin receptors (CMKLRI and CCRL2) were examined in hFOBI.I9 cells after incubation with the osteogenic differentiation medium for nine days. $* P<0.05, * * P<0.01$, compared with control group.

Abbreviations: CMKLRI, chemokine-like receptor I; CCRL2, C-C motif chemokine receptor-like 2.

was associated with risk of osteoporotic fractures. ${ }^{26}$ Conversely, a meta-analysis of 41,738 participants and 5916 patients aged more than 60 years indicated there was a negative correlation between serum 25(OH)D level and the risk of hip fracture, but no association of $25(\mathrm{OH}) \mathrm{D}$ with total fracture risk. ${ }^{3}$ These findings were inconclusive or debatable due to the different bone turnover rates of elderly women.

Chemerin might have an important role in regulating bone metabolism, as demonstrated by a previous study that high chemerin level minimized the bone mass peak, thereby promoting age-related bone loss. ${ }^{23}$ Epidemiological studies verified that chemerin might have a relationship with osteoporosis and $\mathrm{BMD},{ }^{27,28}$ which was also confirmed by our present study that serum chemerin level was inversely correlated to BMD at lumbar spine and femoral neck. Of note, there were conflicting data that serum levels of chemerin were documented to be higher ${ }^{28}$ or lower $^{29}$ in patients with osteoporosis in comparison with control healthy subjects. The reasons for these conflicting results might be explained by the differences in sample size, study population or the influences of either gender or obesity. To minimize this possibility, 

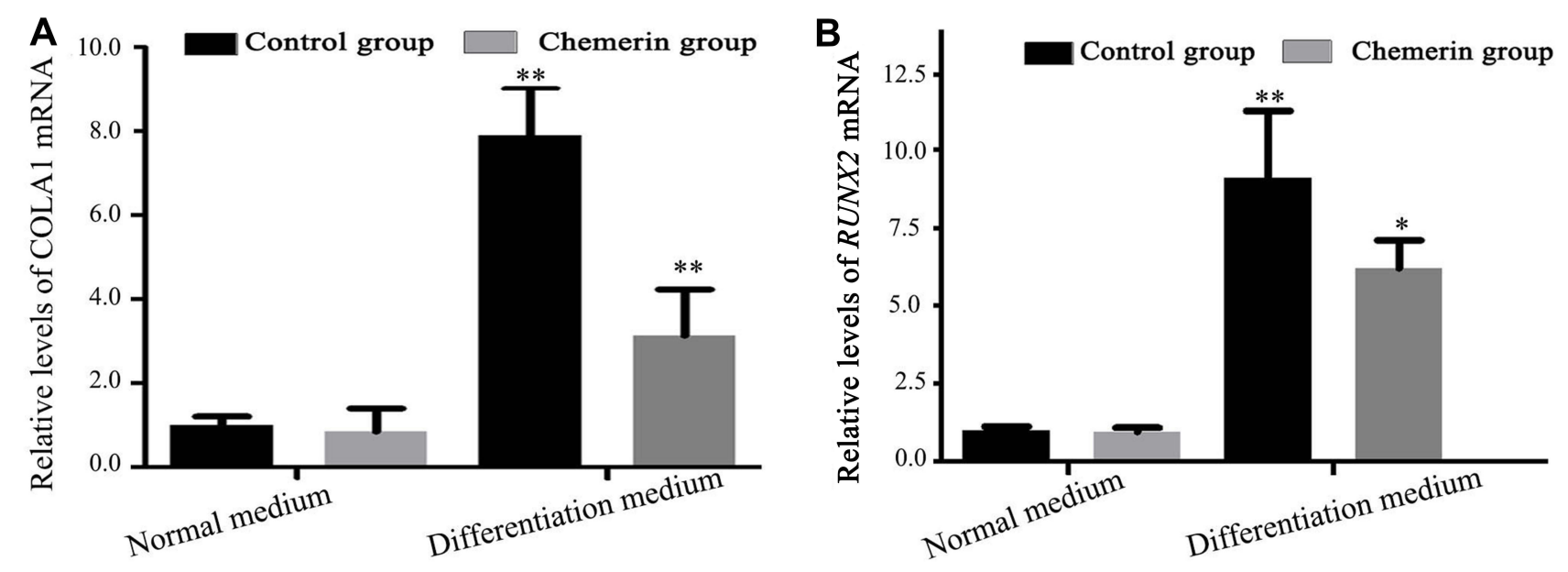

Figure 4 Effect of recombinant human chemerin on the transcription levels of osteoblast differentiation genes.

Notes: hFOBI.19 cells were cultured in osteogenic differentiation medium supplemented with or without $50 \mathrm{ng} / \mathrm{mL}$ recombinant chemerin for nine days and then the transcription levels of COLAI and RUNX2 were examined by qRT-PCR. $* P<0.05$, $* * P<0.01$, compared with normal medium control group.

Abbreviations: CMKLRI, chemokine-like receptor I; CCRL2, C-C motif chemokine receptor like 2.

we adopted an aged- and BMI-matched design, and found that compared with healthy controls, the risk of osteoporotic fracture increased to 2.75-fold higher for subjects with each standard deviation increment of serum chemerin. All these results show a strong association between an increased chemerin level and fracture risk in the elderly Chinese women. In addition, knockdown of chemerin gene in bone marrow stem cells increased the expression of osteoblast marker gene and mineralization, ${ }^{28}$ indicating that chemerin might be a negative regulator during the bone formation. ${ }^{30} \mathrm{~A}$ recent study suggested that there was a positive association between chemerin level and a medium or high risk for osteoporotic fractures in obese men and women of northeastern German population, which may be due to a chemerin-induced negative effect on bone metabolism via abrogation of osteoblastogenesis or stimulation of adipogenesis. ${ }^{31}$ Therefore, it could be speculated that high serum chemerin levels are independently related to stronger inhibitory bone formation and stronger supporting bone resorption.

Chemerin was mainly secreted into the extracellular medium from fat and other tissues. ${ }^{19}$ The secreted chemerin activated CMKLR1, a G protein coupled receptor expressed in various cell types, especially in mesenchymal stem cells, consequently leading to an increased adipogenesis and reduced osteoblastogenesis. ${ }^{17}$ Previous experimental data from rats showed that chemerin and its receptor CMKLR1 might play an important role in osteoblastogenesis, bone mineralization, and inhibition of osteoclastogenesis. ${ }^{28}$ Conversely, stimulation of CCRL2, a non-signaling receptor for chemerin, seemed not to promote any signaling in the cells or induce receptor internalization. Compared with control groups, the transcription levels of $C M K L R 1$ and $C C R L 2$ receptors associated with chemerin did not differ significantly in the hFOB1.19 cells after incubation with osteogenic differentiation medium. Thereby, these chemerin receptors underlying the regulation of bone metabolism still need to be explored in future research.

Bone is a dynamic tissue, which was remodeled continuously through the removal of mineralized bone by osteoclasts, the bone resorbing cells, and the formation of new bone via the osteoblasts, the bone forming cells. ${ }^{18}$ According to the International Federation of Clinical Chemistry and International Osteoporosis Foundation, serum P1NP and $\beta$-CTX levels have been designated as reference standard markers of bone formation and resorption respectively in osteoporosis. In view of the crucial role of BTM, the markers for osteocalcin formation (BAP and P1NP) and for bone resorption ( $\beta$ CTX and NTX) have been confirmed to be independently predictive values of fracture risks. ${ }^{32}$ This was consistent with our results that the serum chemerin level was positively correlated to bone metabolism related markers, such as P1NP, and $\beta$-CTx. In addition, osteoblast differentiation was commonly monitored by a serious of markers and the most frequently used markers were COLA1, ALP, osteopontin, bone sialoprotein, osteocalcin, parathyroid hormone-related protein receptor, and $R U N X 2 .{ }^{33}$ RUNX2 was a master gene for differentiation of mesenchymal stem cells into osteoblasts, ${ }^{29}$ and it reached its highest level in immature osteoblasts, yet decreased during osteoblast maturation. ${ }^{34}$ 

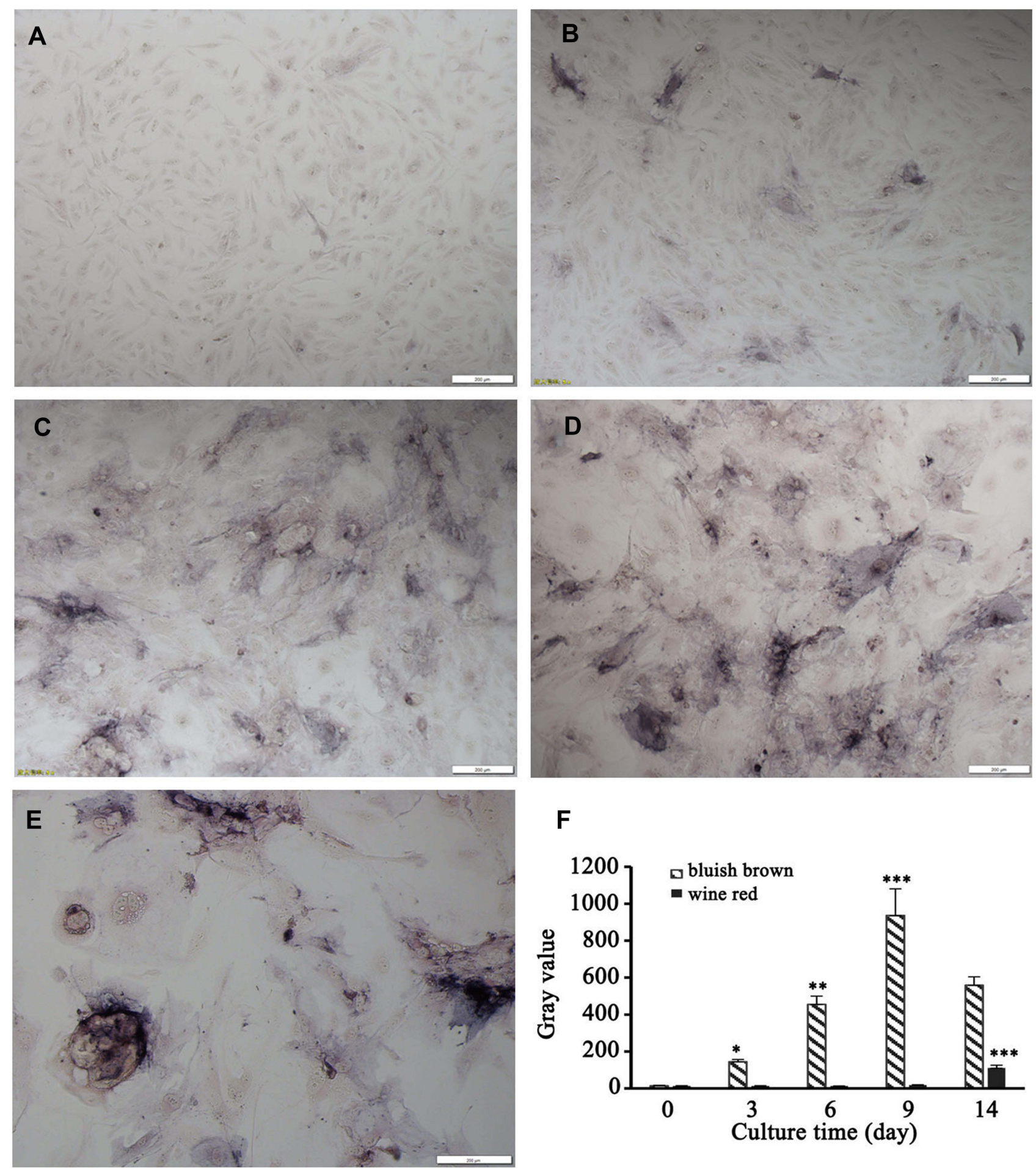

Figure 5 Reprehensive image of hFOBI.19 cells under optical microscope (A-E) and gray values at different culture times (F) (magnification: $40 \times$ ).

Notes: hFOBI.19 cells were collected and harvested for TRACP\&ALP double staining after being incubated with osteogenic differentiation medium supplemented with or without recombinant chemerin for 0 (A), 3 (B), $6(\mathbf{C}), 9$ (D) and 14 (E) days. $* * P<0.0$ I, $* * * P<0.00$ I, compared with control group; $* P<0.05$, compared with other groups.

Our present study verified that the osteoblast marker genes, COLA1 and RUNX2 mRNA, increased significantly during osteogenic differentiation. Notably, a significant decrease of these osteoblast marker genes was detected in the hFOB1.19 cells after incubation with chemerin for nine days post seeding, suggesting that chemerin may inhibit osteoblast maturation via downregulating the transcription level of $R U N X 2$. 
In older age, osteoporosis resulted from the increased bone resorption and decreased bone formation, the rates of which can be evaluated by assaying serum and urinary bone matrix components. ${ }^{35}$ In case both events were coupled and changed in the same direction such as osteoporosis, biomarkers may reflect the overall rate of bone turnover. ${ }^{36}$ Appearance of ALP activity was an early phenotypic marker of osteoblast maturation and synthesis of this enzyme was enhanced along the process of cell differentiation. ${ }^{37}$ Consistently, compared with control group, the results of ALP staining during osteogenic differentiation showed a remarkably decreased number of hFOB1.19 cells with bluish brown after incubation with chemerin for nine days. Interestingly, a number of TRAP-positive cells (wine red) with large volume and multiple nuclei could be observed after incubation with chemerin for 14 days, suggesting that chemerin is of great benefit to promote osteoclast differentiation and maturation. Thus, our study provides an additional evidence that chemerin may have a clinical significance in the identification of elderly women at high risk both of osteoporotic fracture and osteoporosis.

Although we assumed that higher serum chemerin might serve as a risk factor for the patients with osteoporotic fracture, some potential limitations should be noted. We cannot fully exclude the possibility of a reverse causal relationship between serum chemerin and osteoporotic fracture for a limited study population, thus a prospective study with a large number of subjects from different types of population is necessary to supply findings that are more reliable and further to verify the predictive ability of serum chemerin for the osteoporotic fracture. Alternatively, only age- and BMI-matched women were recruited in consideration of some risk factors of serum chemerin, so the samples used in the current study may not be completely representative of the general population for which inferences are required, possibly resulting in selection bias, as well as problems with information bias and confounding. Nevertheless, our current study could provide a basis for future exploration of chemerin on the fracture risk in the elderly women. In the next 5-10 years, it is anticipated that with the innovation of technology, public sharing of scientific data concerning the circulating chemerin will provide a positively influence on the intervention treatment and health care of patients with osteoporotic fracture due to early diagnosis.

\section{Conclusions}

Taken together, our findings establish a key role of osteocyte-expressed chemerin in regulating bone metabolism and further demonstrate a possible mechanism underlying osteoporotic fracture by which osteoblasts and osteocytes control bone formation in the elderly women. However, additional extensive studies of chemerin in bone metabolism should be further explored whether chemerin is qualified as a biomarker for diagnosis of osteoporotic fracture.

\section{Acknowledgments}

This work was supported by the project of Gu Su health talents (GSW2019084) and Kunshan science and technology special project (KS1736).

\section{Author Contributions}

All authors contributed to conception and design, acquisition of data, analysis and interpretation of data; took part in drafting the manuscript or revising it critically; agreed to submit it to the current journal; gave final approval of the version to be published; and agreed to be accounted for all aspects of the work.

\section{Disclosure}

The authors report no conflicts of interest in this work.

\section{References}

1. Paspaliaris V, Kolios G. Stem cells in osteoporosis: from biology to new therapeutic approaches. Stem Cells Int. 2019;2019:1730978. doi:10.1155/ 2019/1730978

2. Ballane G, Cauley JA, Luckey MM, et al. Worldwide prevalence and incidence of osteoporotic vertebral fractures. Osteoporos Int. 2017;2 (5):1531-1542. doi:10.1007/s00198-017-3909-3

3. Wang N, Ji J, Yu S, et al. The relationship between serum vitamin D and fracture risk in the elderly: a meta-analysis. J Orthop Surg Res. 2020;15 (1):81. doi:10.1186/s13018-020-01603-y 
4. Odén A, McCloskey EV, Kanis JA, et al. Burden of high fracture probability worldwide: secular increases 2010-2040. Osteoporos Int. 2015;26 (9):2243-2248. doi:10.1007/s00198-015-3154-6

5. NIH Consensus Development Panel on Osteoporosis Prevention, Diagnosis, and Therapy. Osteoporosis prevention, diagnosis, and therapy. JAMA. 2001;285(6):785-795. doi:10.1001/jama.285.6.785.

6. Aslan A, Karakoyun O, Güler E, Aydin S, Gök MV, Akkurt S. Evaluation of bone mineral density, osteoporosis prevalence and regional risk factors in Turkish women living in Kastamonu: KASTÜRKOS study. Eklem Hastalik Cerrahisi. 2012;23(2):62-67. Turkish. PMID: 22765482.

7. Tremollieres FA, Pouilles JM, Drewniak N, et al. Fracture risk prediction using BMD and clinical risk factors in early postmenopausal women: sensitivity of the WHO FRAX tool. J Bone Miner Res. 2010;25(5):1002-1009. doi:10.1002/jbmr.12

8. Bolland MJ, Siu ATY, Mason BH, et al. Evaluation of the FRAX and Garvan fracture risk calculators in older women. J Bone Miner Res. 2011;26 (2):420-427. doi:10.1002/jbmr.215

9. Cheung CL, Tan KCB, Bow CH, et al. Low handgrip strength is a predictor of osteoporotic fractures: cross-sectional and prospective evidence from the Hong Kong Osteoporosis Study. Age. 2012;34(5):1239-1248. doi:10.1007/s11357-011-9297-2

10. Kim H, Baek KH, Lee SY, et al. Association of circulating dipeptidyl-peptidase 4 levels with osteoporotic fracture in postmenopausal women Osteoporos Int. 2017;28(3):1099-1108. doi:10.1007/s00198-016-3839-5

11. Yang SM, Feskanich D, Willett WC, et al. Association between global biomarkers of oxidative stress and hip fracture in postmenopausal women: a prospective study. J Bone Miner Res. 2014;29(12):2577-2583. doi:10.1002/jbmr.2302

12. Ardawi MSM, Rouzi AA, Al-Sibiani SA, et al. High serum sclerostin predicts the occurrence of osteoporotic fractures in postmenopausal women: the center of excellence for osteoporosis research study. J Bone Miner Res. 2012;27(12):2592-2602. doi:10.1002/jbmr.1718

13. Chen Q, Shou P, Zheng C, et al. Fate decision of mesenchymal stem cells: adipocytes or osteoblasts? Cell Death Differ. 2016;23(7):1128-1139. doi:10.1038/cdd.2015.168

14. Al-Nbaheen M, Vishnubalaji R, Ali D, et al. Human stromal (Mesenchymal) stem cells from bone marrow, adipose tissue and skin exhibit differences in molecular phenotype and differentiation potential. Stem Cell Rev Rep. 2013;9(1):32-43. doi:10.1007/s12015-012-9365-8

15. Shi L, Mao CM, Wang SF, et al. Association of chemerin levels and bone mineral density in Chinese obese postmenopausal women. Medicine. 2016;95(35):e4583. doi:10.1097/MD.0000000000004583

16. Menzel J, Biemann R, Aleksandrova K, et al. The cross-sectional association between chemerin and bone health in peri/pre and postmenopausal women: results from the epic-potsdam study. Menopause. 2017;25(5):574-578. doi:10.1097/GME.0000000000001041

17. Banas M, Zegar A, Kwitniewski M, et al. The expression and regulation of chemerin in the epidermis. PLoS One. 2015;10(2):e0117830. doi:10.1371/journal.pone.0117830

18. De Henau O, Degroot GN, Imbault V, et al. Signaling properties of chemerin receptors CMKLR1, GPR1 and CCRL2. PLoS One. 2016;11(10): e0164179. doi:10.1371/journal.Pone.0164179

19. Terzoudis S, Malliaraki N, Damilakis J, et al. Chemerin, visfatin, and vaspin serum levels in relation to bone mineral density in patients with inflammatory bowel disease. Eur J Gastroenterol Hepatol. 2016;28(7):814-819. doi:10.1097/MEG.0000000000000617

20. Muruganandan S, Dranse HJ, Rourke JL, et al. Chemerin neutralization blocks hematopoietic stem cell osteoclastogenesis. Stem Cells. 2013;31 (10):2172-2182. doi:10.1002/stem.1450

21. Karsenty G. Convergence between bone and energy homeostases: leptin regulation of bone mass. Cell Metab. 2006;4(5):341-348. doi:10.1016/j. cmet.2006.10.008

22. He J, Li JC, Xie H, et al. Serum chemerin levels in relation to osteoporosis and bone mineral density: a case-control study. Dis Markers. 2015;2015:786708. doi:10.1155/2015/786708

23. Kadric L, Zylla S, Nauck M, et al. Associations between plasma chemerin concentrations and bone quality in adults from the general population. Endocrinology. 2018;159(6):2378-2385. doi:10.1210/en.2018-00157

24. Xu L, Mc Elduff P, D’Este C, et al. Does dietary calcium have a protective effect on bone fractures in women? A meta-analysis of observational studies. Br J Nutr. 2004;91(4):625-634. doi:10.1079/BJN20031085

25. Kinyamu HK, Gallagher JC, Prahl JM, et al. Association between intestinal vitamin D receptor, calcium absorption, and serum 1,25 dihydroxyvitamin D in normal young and elderly women. J Bone Miner Res. 1997;12(6):922-928. doi:10.1359/jbmr.1997.12.6.922

26. Zhang L, Chun C, Yang Y, et al. Vitamin D deficiency/insufficiency is associated with risk of osteoporotic thoracolumbar junction vertebral fractures. Med Sci Monit. 2019;25:8260-8268. doi:10.12659/MSM.915780

27. Kanis JA, Mc Closkey EV, Johansson H, et al. A reference standard for the description of osteoporosis. Bone. 2008;42(3):467-475. doi:10.1016/j. bone.2007.11.001

28. Muruganandan S, Roman AA, Sinal CJ. Role of chemerin/CMKLR1 signaling in adipogenesis and osteoblastogenesis of bone marrow stem cells. J Bone Miner Res. 2010;25(2):222-234. doi:10.1359/jbmr.091106

29. Ducy P. Cbfa1: a molecular switch in osteoblast biology. Dev Dyn. 2000;219(4):461-471. doi:10.1002/1097-0177(2000)9999:9999<::AIDDVDY1074>3.0.CO;2-C

30. Liu Y, Song CY, Wu SS, et al. Novel adipokines and bone metabolism. Int J Endocrinol. 2013;2013:895045. doi:10.1155/2013/895045

31. Engin-üstün Y, Çağlayan EK, Göçmen AY, et al. Postmenopausal osteoporosis is associated with serum chemerin and irisin but not with apolipoprotein M levels. J Menopausal Med. 2016;22(2):76-79. doi:10.6118/jmm.2016.22.2.76

32. Mohamed Y, Haifa H, Datel O, et al. The role of biochemical markers of bone turnover in the diagnosis of osteoporosis and predicting fracture risk. Tunis Med. 2014;92(5):304-310. PMID: 25504382.

33. Huang W, Yang S, Shao J, et al. Signaling and transcriptional regulation in osteoblast commitment and differentiation. Front Biosci. 2007;12(812):3068-3092. doi:10.2741/2296

34. Komori T. Runx2, an inducer of osteoblast and chondrocyte differentiation. Histochem Cell Biol. 2018;149(4):313-323. doi:10.1007/s00418-018$1640-6$

35. Atik OŞ, Sezgin EA, Tepedelenlioğlu HE. The role of biomarkers in osteoarthritis and osteoporosis for early diagnosis and monitoring prognosis. Eklem Hastalik Cerrahisi. 2019;30(2):175-176. doi:10.5606/ehc.2019.004

36. Atik OŞ, Aslan A, Odluyurt M. Are fragility fractures being treated properly? Jt Dis Relat Surg. 2020;31(2):403-404. doi:10.5606/ehc.2020.57894

37. Marom R, Shur I, Solomon R, et al. Characterization of adhesion and differentiation markers of osteogenic marrow stromal cells. $J$ Cell Physiol. 2005;202(1):41-48. doi:10.1002/jcp.20109 


\section{Publish your work in this journal}

The International Journal of Women's Health is an international, peer-reviewed open-access journal publishing original research, reports, editorials, reviews and commentaries on all aspects of women's healthcare including gynecology, obstetrics, and breast cancer. The manuscript management system is completely online and includes a very quick and fair peer-review system, which is all easy to use. Visit http://www. dovepress.com/testimonials.php to read real quotes from published authors.

Submit your manuscript here: https://www.dovepress.com/international-journal-of-womens-health-journal 